\title{
Two-Stage Revision Using a Modified Articulating Spacer in Infected Total Knee Arthroplasty
}

\author{
Young Soo Kim, MD, Ki Cheor Bae, MD, Chul Hyun Cho, MD, Kyung Jae Lee, MD, Eun Seok Sohn, MD, and \\ Beom Soo Kim, MD \\ Department of Orthopedic Surgery, Keimyung University School of Medicine, Daegu, Korea
}

Purpose: To evaluate clinical results of two-stage revision using a modified articulating spacer for treatment of infected total knee arthroplasty (TKA). Materials and Methods: We retrospectively reviewed 20 cases treated by two-stage revision arthroplasty using a modified articulating spacer under the diagnosis of infected TKA from January 2006 to December 2011. The mean follow-up period was 22.3 months. The first operation consisted of debridement after removal of the prosthesis, reinsertion of the femoral component after autoclaving, and implantation of antibiotic-loaded cement with a new polyethylene in the proximal tibia.

Results: The mean period between the primary TKA and the first stage operation was 39 months and between the first stage operation and the revision arthroplasty was 3.3 months. The average range of motion (ROM) increased from $69.8^{\circ}$ preoperatively to $102.8^{\circ}$ postoperatively ( $<<0.001$ ). The mean Knee Society knee score increased from 33.8 points to 85.3 points ( $\mathrm{p}<0.001$ ). The mean Knee Society function score increased from 35 points to 87.5 points $(\mathrm{p}<0.001)$. The mean Hospital for Special Surgery score increased from 57.6 points preoperatively to 82.6 points postoperatively $(\mathrm{p}<0.001)$. Two cases $(10 \%)$ were re-infected after the revision arthroplasty.

Conclusions: Two-stage revision arthroplasty using an articulating cement spacer can be an effective therapy not only for the treatment of an infected TKA but also for recovery of knee ROM and function.

Keywords: Knee, Infection, Revision total knee arthroplasty, Two-stage

\section{Introduction}

Infection is one of the major complications of total knee arthroplasty (TKA) that can be best treated with two-stage revision arthroplasty ${ }^{1,2)}$. However, the use of non-articulating spacers, such as bead spacers and static block spacers, in two-stage revision has been associated with undesirable side effects: the difficulty of joint exposure at the time of revision arthroplasty due to scarring and shortening of the joint capsule and quadriceps, additional

Received July 16, 2012; Revised (1st) May 14, 2013; (2nd) July 8, 2013; (3rd) September 15, 2013; Accepted October 22, 2013

Correspondence to: Ki Cheor Bae, MD

Department of Orthopedic Surgery, Keimyung University School of

Medicine, 56 Dalseong-ro, Jung-gu, Daegu 700-712, Korea

Tel: +82-53-250-7729, Fax: +82-53-250-7205

E-mail: bkc@dsmc.or.kr

This is an Open Access article distributed under the terms of the Creative Commons Attribution Non-Commercial License (http://creativecommons.org/licenses/by-nc/3.0/) which permits unrestricted non-commercial use, distribution, and reproduction in any medium, provided the original work is properly cited. bone loss, and impaired postoperative rehabilitation and functional recovery due to restriction on weight-bearing between the two stages ${ }^{3)}$.

The introduction of articulating prosthesis with antibioticloaded acrylic cement (PROSTALAC; DePuy Orthopaedics Inc., Warsaw, IN, USA) has contributed to reduced infection rates and increased range of motion (ROM). Unfortunately, it is neither affordable nor applicable to all patients ${ }^{4,5)}$. Thus, some studies suggested using articulating prostheses that are manufactured intraoperatively by the surgeon ${ }^{6,7)}$. The custom-made spacers are described as facilitating the procedure, allowing for early ambulation and minimal soft tissue contractures, and yielding favorable outcomes in terms of preservation of the muscle strength and ROM. Still, prolonged use of such prostheses has been associated with quadriceps contractures and joint capsule adhesion in twostage revision. Hence, efforts have been focused on inventing temporary articulating spacers for two-stage revision arthroplasty. In this article, we present our experience of two-stage revision TKA using a modified articulating spacer that was effective for facilitating joint mobility and eradicating infection with a review 

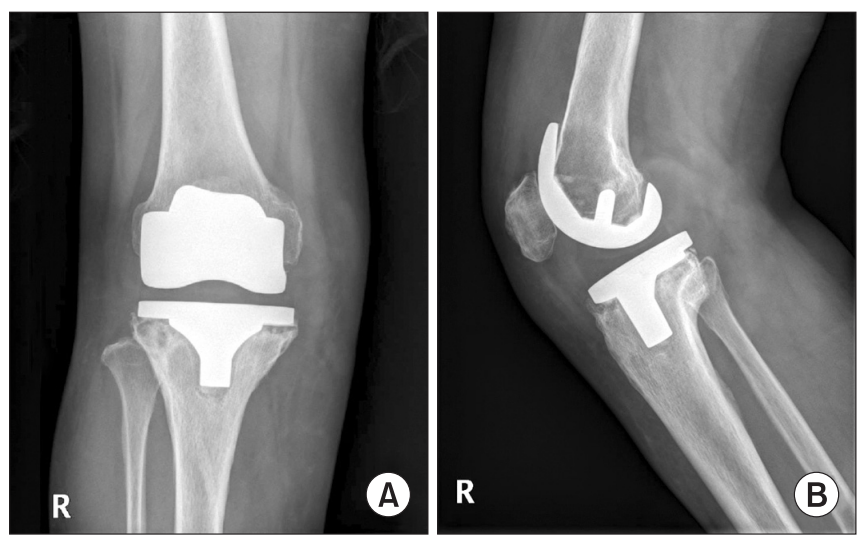

Fig. 1. A 56-year-old woman who received primary total knee arthroplasty (TKA) due to osteoarthritis complained of persistent pain and diffuse swelling after TKA. At 27 months after primary TKA, the radiographs show radiolucency around the tibial prosthesis.

of the literature. The surgery consisted of reinsertion of the autoclaved femoral component and implantation of a new polyethylene with antibiotic-loaded cement in the proximal tibia.

\section{Materials and Methods}

\section{Study Population}

Of the patients who were treated for infected TKA between January 2006 and December 2011, 20 patients (20 knees) who underwent two-stage revision arthroplasty using a modified articulating spacer were included in this study. Fifteen patients had undergone primary TKA at our institution and the remaining 5 patients were transferred from other institutions after having been diagnosed with infection after TKA. Infection was diagnosed based on blood test results including white blood cell (WBC) count, erythrocyte sedimentation rate (ESR), and C-reactive protein (CRP) level, radiographic findings (Fig. 1), and bacterial culture results. There were 5 males and 15 females and their mean age at the time of primary TKA was 61.5 years (range, 36 to 75 years). The mean follow-up period was 22.3 months (range, 14 to 60 months). The indications for surgery were degenerative arthritis in 16 patients and rheumatoid arthritis in 4 patients.

\section{Operative Technique}

A skin incision was made over the previous incision line created during the primary TKA and the knee joint was exposed using a medial parapatellar approach. The knee prosthesis and bone cement were removed and thorough debridement was performed. Inflammatory tissues obtained during this procedure were sent for gram staining, aerobic and anaerobic bacterial culture, fungal

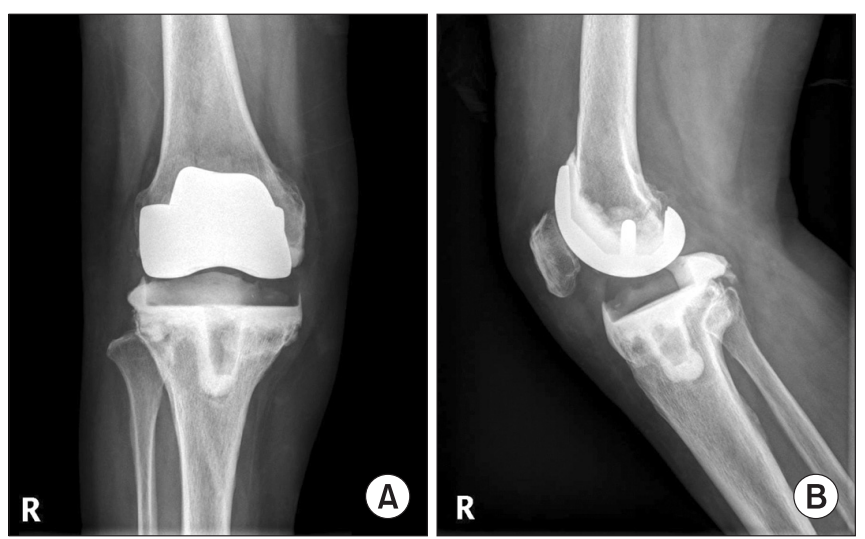

Fig. 2. (A) Anteroposterior and (B) lateral radiographs obtained after the first stage operation using a modified articulating spacer.

culture, and acid fast bacilli staining and culture.

The removed femoral component was cleaned of bone cement and debris, washed, and sterilized in an autoclave for 7 minutes at $137^{\circ} \mathrm{C}$ in the operating room. Meanwhile, joint irrigation, removal of the remaining bone cement and necrotic debris, and synovectomy were performed. For bone cementing, if the preoperative culture was negative, $4 \mathrm{~g}$ of vancomycin was mixed with CMW 3 (Blue Cross, DePuy Orthopaedics Inc.) bone cement containing 1 $\mathrm{g}$ of gentamycin sulphate per $40 \mathrm{~g}$ packet of cement. Otherwise, a mixture of cephalosporin antibiotics sensitive to the cultured organism was used. In the tibia, the antibiotic-loaded bone cement was applied without molding and a new polyethylene insert was placed not to cause shift of the joint line from primary TKA. The new polyethylene insert was thicker than that used in the primary surgery to compensate for the removal of infected bone tissues and was mobile bearing for implantation with bone cement in the tibia. Joint stability was assessed with the knee in flexion and extension (Fig. 2). Joint exercises using a continuous passive motion machine were initiated within one to two days after the first stage procedure. From the fifth to sixth postoperative day, partial weight-bearing with a walker was started. The Lenox hill derotation orthosis was prescribed to wear for patients complaining of discomfort during walking (15 patients, $75 \%$ ).

\section{Infection Treatment}

The total duration of antibiotic therapy was 10 weeks. Antibiotics were administered intravenously for 6 weeks after the first stage surgery. The intravenous antibiotic treatment was discontinued if the blood test performed once per week shows normal WBC count, ESR, and CRP level, joint aspirate culture is negative at the 6th postoperative week, and symptoms of inflammation, such as swelling, heat sensation, and tenderness, are not present. 


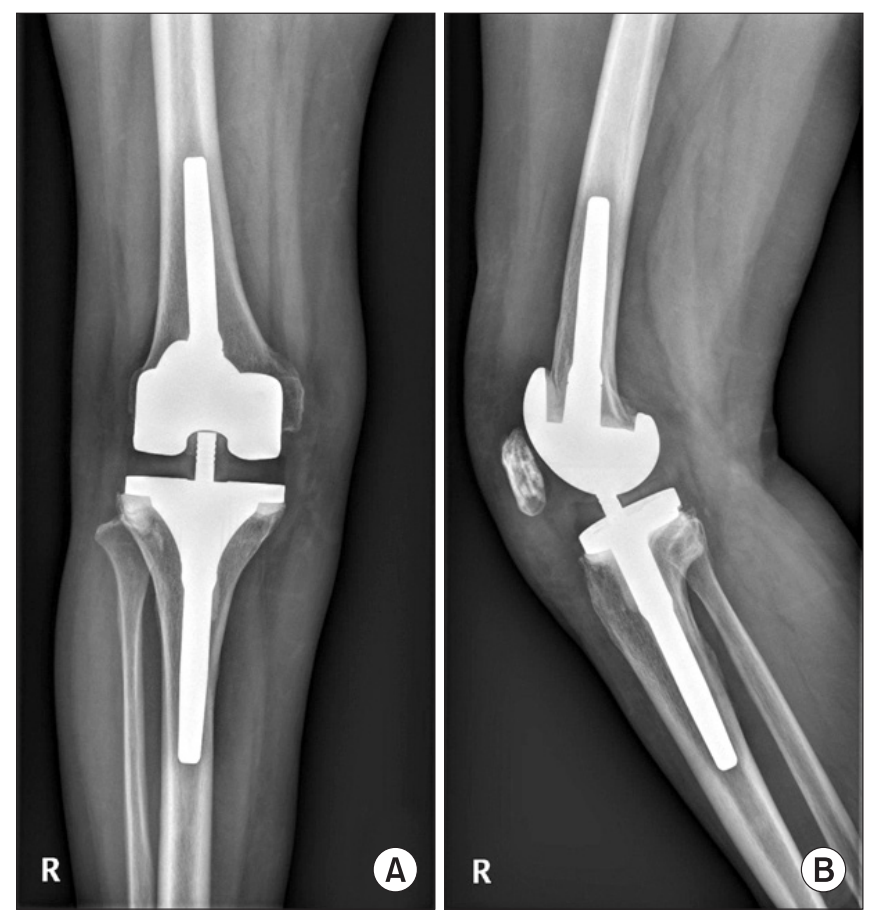

Fig. 3. (A) Anteroposterior and (B) lateral radiographs obtained at 18 months after second-stage revision arthoplasty using a stemmed prosthesis.

Subsequently, four weeks of oral antibiotic treatment was started and joint aspiration was performed at the end of the treatment. Patients were followed without further therapy for the following 2 weeks until another joint aspiration was done. The blood test results were normal and the aspirate culture was negative in all patients during the period when the antibiotics were withdrawn. Thus, a second stage surgery was scheduled for all patients. Since the intraoperative frozen section examination revealed less than five WBCs per high power field in all patients, the second stage procedure was performed as planned (Fig. 3).

\section{Clinical Assessment}

The postoperative ROM, Knee Society knee score (KSKS), Knee Society function score (KSFS), Hospital for Special Surgery (HSS) score, and recurrence of infection were assessed. Statistical analysis was performed using SPSS ver. 12.0 (SPSS Inc., Chicago, IL, USA). A paired T-test was used for all statistical analysis with $\mathrm{p}<0.05$ considered statistically significant.

\section{Results}

\section{Microbiological Results}

Of the 20 patients, the culture was positive in 16 patients (80\%) and negative in 4 patients (20\%). The organisms identified from preoperative and intraoperative cultures were Methicillin-resistant Staphylococcus epidermidis in 7 patients, Methicillin-resistant Staphylococcus aureus in 4 patients, Methicillin-sensitive Staphylococcus aureus in 3 patients, and Enterococcus feacalis (E. feacalis) in 2 patients (Table 1).

\section{Blood Test Results}

The mean WBC count decreased from $14,580 / \mu \mathrm{L}$ (range, 10,400 to $24,240 \mu \mathrm{L}$ ) prior to first stage surgery to $7.830 / \mu \mathrm{L}$ (range, 4,300 to $8,540 \mu \mathrm{L}$ ) prior to second stage surgery. The mean ESR decreased from $54.11 \mathrm{~mm} / \mathrm{hr}$ (range, 48 to $82 \mathrm{~mm} /$ hr) prior to first stage surgery to $23.41 \mathrm{~mm} / \mathrm{hr}$ (range, 12 to 28 $\mathrm{mm} / \mathrm{hr}$ ) prior to second stage surgery. The mean CRP level decreased from $6.98 \mathrm{mg} / \mathrm{dL}$ (range, 1.31 to $14 \mathrm{mg} / \mathrm{dL}$ ) prior to first stage surgery to $0.39 \mathrm{mg} / \mathrm{dL}$ (range, 0.05 to $0.48 \mathrm{mg} / \mathrm{dL}$ ) prior to second stage surgery.

\section{Clinical Results}

The mean interval from the onset of symptoms of infection following TAK to first stage surgery was 39 months (range, 24 to 77 months) and the mean interval from first stage surgery to second stage surgery was 3.3 months (range, 3 to 4 months). The 16 patients with positive cultures after the first stage surgery were treated with intravenous antibiotics sensitive to the cultured organisms. In the remaining 4 patients with negative cultures, cephalosporin class antibiotics were administered intravenously and orally.

The mean ROM was increased from $69.8^{\circ}$ (range, $50^{\circ}$ to $100^{\circ}$ ) prior to first stage surgery to $98^{\circ}$ (range, $70^{\circ}$ to $115^{\circ}$ ) prior to second stage surgery, and then to $102.8^{\circ}$ (range, $80^{\circ}$ to $130^{\circ}$ ) following second stage surgery $(\mathrm{p}<0.001)$. The mean KSKS increased from 33.8 points (range, 28 to 52 points) prior to first stage surgery to 85.3 points (range, 77 to 94 points) following second stage surgery $(\mathrm{p}<0.001)$. The mean KSFS increased from 35.0 points (range, 20 to 55 points) prior to first stage surgery to 87.5 points (range, 70 to 100 points) following second stage surgery $(\mathrm{p}<0.001)$. The mean HSS score increased from 57.6 points (range, 27 to 80 points) prior to first stage surgery to 82.6 points (range, 56 to 100 points) following second stage surgery $(\mathrm{p}<0.001)$. Partial weightbearing was permitted at a mean of 5.6 days (range, 5 to 6 days) after first stage surgery. There was no sign of infection recurrence in 18 patients until the last follow-up based on the clinical examination and blood test results; however, extended period of antibiotic therapy and revision surgery using an articulating cement spacer were required in 2 patients $(10 \%)$ due to recurrent 
Table 1. Clinical Data of the Patients

\begin{tabular}{|c|c|c|c|c|c|c|c|c|}
\hline Case & Age (yr) & Sex & Diagnosis & Cultured organism & $\operatorname{ROM}\left(^{\circ}\right)$ & HSS score & KSKS & KSFS \\
\hline 1 & 71 & $\mathrm{~F}$ & $\mathrm{OA}$ & MRSE & $50 / 90$ & $27 / 56$ & $28 / 87$ & $20 / 90$ \\
\hline 2 & 75 & $\mathrm{~F}$ & RA & $(-)$ & $60 / 110$ & $50 / 80$ & $38 / 88$ & $55 / 90$ \\
\hline 3 & 51 & $\mathrm{~F}$ & OA & MRSE & $55 / 95$ & $45 / 75$ & $38 / 78$ & $45 / 80$ \\
\hline 4 & 69 & $\mathrm{~F}$ & $\mathrm{OA}$ & MRSE & $75 / 115$ & $60 / 80$ & $40 / 82$ & $35 / 90$ \\
\hline 5 & 54 & $\mathrm{~F}$ & OA & MRSA & $80 / 125$ & $65 / 90$ & $52 / 84$ & $50 / 90$ \\
\hline 6 & 58 & $\mathrm{~F}$ & RA & MSSA & $90 / 130$ & $70 / 100$ & $28 / 80$ & $30 / 80$ \\
\hline 7 & 36 & $\mathrm{~F}$ & RA & MRSE & $50 / 80$ & $30 / 85$ & $28 / 85$ & $30 / 100$ \\
\hline 8 & 59 & $\mathrm{~F}$ & OA & $(-)$ & $65 / 90$ & $55 / 70$ & $32 / 87$ & $35 / 90$ \\
\hline 9 & 65 & $\mathrm{~F}$ & OA & MRSE & $75 / 100$ & $65 / 85$ & $28 / 88$ & $35 / 100$ \\
\hline 10 & 57 & M & OA & MSSA & $50 / 90$ & $35 / 75$ & $32 / 85$ & $30 / 90$ \\
\hline 11 & 63 & $\mathrm{~F}$ & OA & MSSA & $70 / 100$ & $60 / 80$ & $28 / 89$ & $30 / 90$ \\
\hline 12 & 69 & $\mathrm{~F}$ & OA & MRSA & $65 / 95$ & $55 / 75$ & $40 / 87$ & $35 / 80$ \\
\hline 13 & 64 & M & OA & MRSA & $75 / 95$ & $60 / 75$ & $32 / 89$ & $40 / 90$ \\
\hline 14 & 49 & $\mathrm{~F}$ & RA & E. feacalis & $80 / 95$ & $65 / 80$ & $40 / 82$ & $45 / 90$ \\
\hline 15 & 71 & M & OA & MRSE & $75 / 95$ & $70 / 75$ & $28 / 81$ & $40 / 90$ \\
\hline 16 & 69 & $\mathrm{~F}$ & OA & $(-)$ & $90 / 100$ & $75 / 90$ & $30 / 86$ & $30 / 70$ \\
\hline 17 & 64 & M & OA & $(-)$ & $50 / 105$ & $55 / 90$ & $30 / 77$ & $30 / 80$ \\
\hline 18 & 49 & M & OA & E. feacalis & $55 / 105$ & $55 / 90$ & $40 / 86$ & $30 / 90$ \\
\hline 19 & 71 & M & OA & MRSA & $85 / 110$ & $75 / 100$ & $32 / 94$ & $25 / 80$ \\
\hline 20 & 59 & $\mathrm{~F}$ & OA & MRSE & $100 / 130$ & $80 / 100$ & $32 / 90$ & $30 / 90$ \\
\hline Mean & 61.5 & & & & $69.75 / 102.75$ & $57.6 / 82.55$ & $33.8 / 85.25$ & $35 / 87.5$ \\
\hline
\end{tabular}

Values are presented as 'before first operation/last follow-up'.

ROM: range of motion, HSS: Hospital for Special Surgery, KSKS: Knee Society knee score, KSFS: Knee Society function score, OA: osteoarthritis, MRSE: methicillin-resistant Staphylococcus epidermidis, RA: rheumatoid arthritis, MSSA: methicillin-sensitive Staphylococcus aureus, E. feacalis: Enterococcus feacalis.

infection. Although diabetes mellitus was present in the latter 2 patients, 9 of the former 18 patients (50\%) without recurrent infection were also diagnosed with the disease, indicating there is no statistically significant relationship between the disease and relapse of infection ( $\mathrm{p}=0.494)$. Complications, such as medial collateral ligament tears and periprosthetic fractures, were not observed in any of the patients.

\section{Discussion}

Treatment of infection following TKA has been the focus of numerous clinical studies that suggest treatment options, such as antibiotic therapy, debridement, resection arthroplasty, knee arthrodesis, revision arthroplasty, and limb amputation ${ }^{1,3,-11)}$.

Currently, two-stage revision arthroplasty is widely performed for the treatment of infected TKA. The two-stage procedure consists of removal of knee components followed by intravenous an- tibiotic treatment and use of antibiotic-loaded bone cement to increase the concentration of antibiotics at the infection site ${ }^{3,10,12,13)}$. In particular, articulating cement spacers are preferred in twostage revisions for prevention of joint contractures and retention of ROM. Compared to non-articulating ones, the articulating spacers provide better functional outcomes by enabling early rehabilitation and ROM exercises between the two stages and make soft tissue resection easier during the second stage procedure ${ }^{14)}$.

Although implants designed for two-stage revision arthroplasty, such as the PROSTALAC system (DePuy Orthopaedics Inc.), have been introduced, they are neither affordable nor applicable to all patients ${ }^{2,3)}$. Hence, the procedure is mostly performed by using an articulating spacer custom-made by the surgeon during surgery. Techniques for manufacturing personalized articulating cement spacers for two-stage TKA are well described by Hoffmann et al. ${ }^{14)}$, Fehring et al. ${ }^{2}$, and Goldstein et al. ${ }^{15)}$ and satisfactory results of two-stage revision using custom-made articulating 
cement spacers have been documented in some domestic studies by Bae and $\mathrm{Yim}^{3)}$, Kim et al. ${ }^{16)}$, and Ha and $\mathrm{Awe}^{17)}$.

The technique we used among our patients is similar to that described by Kim et al. ${ }^{16}$, but it can be differentiated in terms of the use of a new polyethylene insert. A failure to maintain an appropriate joint space between the two stages due to bone defect and collateral ligament insufficiency in the infected knee increases the risk of dislocation or instability of the implanted antibioticloaded cement spacer ${ }^{18}$. Thus, we took care to lower the risk of joint instability by maintaining the thickness of the previous tibial component with use of antibiotic-loaded cement and inserting a thicker polyethylene than that used in the primary TKA. The advantages of our technique include 1) reduced cost due to the reuse of the femoral component after autoclaving, 2) prevention of autoclaving-related changes in the morphological and functional properties of the polyethylene insert, and 3) customization of the polyethylene insert size according to the extent of bone loss. The instability and flexion/extension of the knee were assessed with the polyethylene insert in place. The relatively delicate procedure for manufacturing a femoral component could be avoided by reusing the component that had been placed in the primary surgery after autoclaving, which resulted in reduced operating time. Any joint instability could be managed by adjusting the thickness of the polyethylene insert.

The timing of reimplantation is crucial to treatment success of two-stage revision ${ }^{19)}$. Insall et al. ${ }^{20)}$ suggested that a second stage surgery should be performed when soft tissue is in good condition and joint aspiration shows no evidence of infection. Mont et al. ${ }^{21)}$ advocated the importance of preoperative evaluation of cultures and sensitivity through biopsy and joint aspiration. Regarding the period of antibiotic therapy, Mahmud et al. ${ }^{22)}$ proposed at least 6 weeks of intravenous antibiotic injection prior to reimplantation. In our study, we assessed clinical symptoms and soft tissue condition before proceeding with the second stage surgery. Joint aspiration was performed after 6 weeks of intravenous antibiotic injection following the first stage surgery. Oral antibiotics were given for the following 4 weeks. The antibiotic treatment was withdrawn for the subsequent 2 weeks and three separate joint aspirations were performed. If the aspirate cultures were negative, it was determined to proceed with reimplantation. The mean interval between the two stages was 3.3 months.

Reimplantation was performed taking care to restore the closest to the preoperative joint condition to facilitate postoperative ambulation and rehabilitation. The mean interval between the first stage surgery and initiation of partial weight-bearing was 5.6 days (range, 5 to 6 days), indicating relatively early ambulation was possible. Early ambulation increased patient satisfaction in performing daily living activities, such as using the bathroom without help and walking without aid, and thus patient compliance was high until the second stage surgery.

The mean ROM was significantly improved from $69.8^{\circ}$ preoperatively to $98^{\circ}$ prior to second stage surgery, and then to $102.8^{\circ}$ after reimplantation, indicating that the two-stage revision using a modified articulating cement spacer was effective for improving $\mathrm{ROM}$ and functional recovery $(\mathrm{p}<0.001)$. Statistically significant improvements were also noted in the mean KSKS (from 33.8 prior to first stage surgery to 85.3 after reimplantation, $\mathrm{p}<0.001$ ) and the mean KSFS (from 35.0 prior to first stage surgery to 87.5 after reimplantation, $\mathrm{p}<0.001)$. The mean HSS score remarkably increased $(\mathrm{p}<0.001)$ during the same period. We believe these results demonstrate the efficacy of the two-stage revision arthroplasty using a modified articulating spacer.

Infection recurred in two of the 20 patients. During the followup after reimplantation, the two patients presented with pain, localized heat, and increased WBC count, ESR, and CRP level, which were considered as evidence of infection, and thus repetitive debridement and intravenous antibiotic injection were performed. In spite of this, the symptoms did not subside, which led us to remove the femoral component and polyethylene insert with cement that were implanted in the first stage surgery and perform another second stage surgery to manage the infection.

The two-stage revision arthroplasty using a modified articulating cement spacer provided successful results in 18 of the 20 patients. However, the limitations of our study include that there was no comparison with TKAs using non-articulating spacers and the number of cases was small.

Two-stage revision arthroplasty using a modified articulating cement spacer can be a reliable and effective procedure for the treatment of infected TKA. The procedure improved patient function and compliance with the treatment and facilitated recovery of ROM and joint function by providing joint stability and mobility during the interval treatment period and yielded a high infection eradication rate.

\section{Conclusions}

Two-stage revision arthroplasty using a modified articulating cement spacer can be useful for the treatment of infected TKA in terms of infection eradication and recovery of joint mobility and function. 


\section{Conflict of Interest}

No potential conflict of interest relevant to this article was reported.

\section{References}

1. Borden LS, Gearen PF. Infected total knee arthroplasty: a protocol for management. J Arthroplasty. 1987;2:27-36.

2. Fehring TK, Odum S, Calton TF, Mason JB. Articulating versus static spacers in revision total knee arthroplasty for sepsis. The Ranawat Award. Clin Orthop Relat Res. 2000;(380):9-16.

3. Bae DK, Yim C. Role of articulating spacer in two stage reimplantation of infected total knee arthroplasty. J Korean Knee Soc. 1998;10:154-60.

4. Meek RM, Masri BA, Dunlop D, Garbuz DS, Greidanus NV, McGraw R, Duncan CP. Patient satisfaction and functional status after treatment of infection at the site of a total knee arthroplasty with use of the PROSTALAC articulating spacer. J Bone Joint Surg Am. 2003;85:1888-92.

5. Haddad FS, Masri BA, Campbell D, McGraw RW, Beauchamp CP, Duncan CP. The PROSTALAC functional spacer in two-stage revision for infected knee replacements. Prosthesis of antibiotic-loaded acrylic cement. J Bone Joint Surg Br. 2000;82:807-12.

6. Masri BA, Kendall RW, Duncan CP, Beauchamp CP, McGraw RW, Bora B. Two-stage exchange arthroplasty using a functional antibiotic-loaded spacer in the treatment of the infected knee replacement: the Vancouver experience. Semin Arthroplasty. 1994;5:122-36.

7. Hsu YC, Cheng HC, Ng TP, Chiu KY. Antibiotic-loaded cement articulating spacer for 2-stage reimplantation in infected total knee arthroplasty: a simple and economic method. J Arthroplasty. 2007;22:1060-6.

8. Ocguder A, Firat A, Tecimel O, Solak S, Bozkurt M. Twostage total infected knee arthroplasty treatment with articulating cement spacer. Arch Orthop Trauma Surg. 2010;130:719-25.

9. Cho WS, Jeong YG, Park JH, Shin HK, Kim KY, Seon MW. Treatment of infected TKRA. J Korean Orthop Assoc. 2001;36:561-7.

10. Lee DY, Shim JI, Kim TS, Lee SJ, Lee SH, Lee DK, Yoo YS, Han YH. Two-stage reimplantation for the treatment of infected total knee arthroplasty. J Korean Orthop Assoc. 1999;34:319-25.

11. Rand JA, Fitzgerald RH Jr. Diagnosis and management of the infected total knee arthroplasty. Orthop Clin North Am. 1989;20:201-10.

12. Windsor RE, Bono JV. Infected total knee replacements. J Am Acad Orthop Surg. 1994;2:44-53.

13. Windsor RE, Insall JN, Urs WK, Miller DV, Brause BD. Twostage reimplantation for the salvage of total knee arthroplasty complicated by infection: further follow-up and refinement of indications. J Bone Joint Surg Am. 1990;72:272-8.

14. Hofmann AA, Kane KR, Tkach TK, Plaster RL, Camargo MP. Treatment of infected total knee arthroplasty using an articulating spacer. Clin Orthop Relat Res. 1995;(321):45-54.

15. Goldstein WM, Kopplin M, Wall R, Berland K. Temporary articulating methylmethacrylate antibiotic spacer (TAMMAS): a new method of intraoperative manufacturing of a custom articulating spacer. J Bone Joint Surg Am. 2001;83 Suppl 2 Pt 2:92-7.

16. Kim SH, Han HS, Kim DH, Kang SB. Two-stage reimplantation in infected total knee arthroplasty (a method of reinsertion of the autoclaved femoral component and a polyethylene liner). J Korean Knee Soc. 2010;22:110-6.

17. Ha CW, Awe SI. Two stage revision using custom articulating spacer in an infected TKA: a novel method for an intraoperative construction of an articulating spacer. J Korean Orthop Assoc. 2005;40:409-17.

18. Pietsch M, Hofmann S, Wenisch C. Treatment of deep infection of total knee arthroplasty using a two-stage procedure. Oper Orthop Traumatol. 2006;18:66-87.

19. Burnett RS, Kelly MA, Hanssen AD, Barrack RL. Technique and timing of two-stage exchange for infection in TKA. Clin Orthop Relat Res. 2007;464:164-78.

20. Insall JN, Thompson FM, Brause BD. Two-stage reimplantation for the salvage of infected total knee arthroplasty. J Bone Joint Surg Am. 1983;65:1087-98.

21. Mont MA, Waldman BJ, Hungerford DS. Evaluation of preoperative cultures before second-stage reimplantation of a total knee prosthesis complicated by infection: a comparison-group study. J Bone Joint Surg Am. 2000;82:1552-7.

22. Mahmud T, Lyons MC, Naudie DD, Macdonald SJ, McCalden RW. Assessing the gold standard: a review of 253 two-stage revisions for infected TKA. Clin Orthop Relat Res. 2012;470:2730-6. 